\title{
Animal Models in Studies of Cardiotoxicity Side Effects from Antiblastic Drugs in Patients and Occupational Exposed Workers
}

\author{
Monica Lamberti, ${ }^{1}$ Giancarlo Giovane, ${ }^{1}$ Elpidio M. Garzillo, ${ }^{1}$ Franca Avino, ${ }^{2}$ \\ Antonia Feola, ${ }^{3}$ Stefania Porto, ${ }^{3}$ Vincenzo Tombolini, ${ }^{4,5}$ and Marina Di Domenico ${ }^{3,6}$ \\ ${ }^{1}$ Department of Experimental Medicine, Section of Hygiene, Occupational Medicine and Forensic Medicine, \\ Area of Occupational Medicine, Second University of Naples, Via L. De Crecchio 7, 80138 Naples, Italy \\ ${ }^{2}$ National Institute for the Study and Treatment of Cancer, Foundation "G. Pascale", Via Mariano Semmola, 80131 Napoli, Italy \\ ${ }^{3}$ Department of Biochemistry, Biophysics and General Pathology, Second University of Naples, Via L. De Crecchio 7, 80138 Naples, Italy \\ ${ }^{4}$ Department of Radiology, Oncology and Pathological Anatomy Sciences, University of Rome, "La Sapienza", \\ Piazzale Aldo Moro 5, 00185 Rome, Italy \\ ${ }^{5}$ Spencer-Lorillard Foundation, University of Rome, "La Sapienza", Piazzale Aldo Moro 5, 00185 Rome, Italy \\ ${ }^{6}$ Sbarro Institute for Cancer Research and Molecular Medicine, Center for Biotechnology, Building Suite 431, \\ 1900 North 12th Street, Philadelphia, PA 19122, USA
}

Correspondence should be addressed to Marina Di Domenico; marina.didomenico@unina2.it

Received 31 July 2013; Revised 29 October 2013; Accepted 7 November 2013; Published 19 February 2014

Academic Editor: Monica Fedele

Copyright (C) 2014 Monica Lamberti et al. This is an open access article distributed under the Creative Commons Attribution License, which permits unrestricted use, distribution, and reproduction in any medium, provided the original work is properly cited.

Cardiotoxicity is an important side effect of cytotoxic drugs and may be a risk factor of long-term morbidity for both patients during therapy and also for staff exposed during the phases of manipulation of antiblastic drugs. The mechanism of cardiotoxicity studied in vitro and in vivo essentially concerns the formation of free radicals leading to oxidative stress, with apoptosis of cardiac cells or immunologic reactions, but other mechanisms may play a role in antiblastic-induced cardiotoxicity. Actually, some new cytotoxic drugs like trastuzumab and cyclopentenyl cytosine show cardiotoxic effects. In this report we discuss the different mechanisms of cardiotoxicity induced by antiblastic drugs assessed using animal models.

\section{Introduction}

Many anticancer drugs have, as side effect, the risk of severe cardiotoxicity by a cumulative, dose-dependent toxicity for both patients during therapy and also for healthcare workers during the phases of manipulation of antiblastic drugs (Table 1). In fact, several scientific studies have shown that in exposed workers the presence of several cardiotoxic drugs like doxorubicin, epirubicin, cyclophosphamide, and 5 -fluourouracil was often identified in urine $[1,2]$. Cardiotoxicity effects include small changes in blood pressure as well as arrhythmias and cardiomyopathy [3]. Mechanisms of cardiotoxicity by antiblastic drugs comprise cellular damage, with the formation of free oxygen radicals and the induction of immunogenic reactions with the presence of antigen presenting cells in the heart [4]. Early and late onset cardiac effects are reported; the first effect can be acute, subacute, or chronically progressive [5]. Acute or subacute cardiotoxicity effects of antiblastic drugs are rare; they occur during or immediately following infusion and are usually transient (e.g., electrocardiographic abnormalities such as nonspecific ST-T changes and QT prolongation, pericarditis-myocarditis syndrome, and ventricular dysfunction with congestive heart failure) [6]. The late effect generally starts within one year after the beginning of antiblastic therapy with chronic cardiac abnormalities and can progress to overt cardiac disease. 


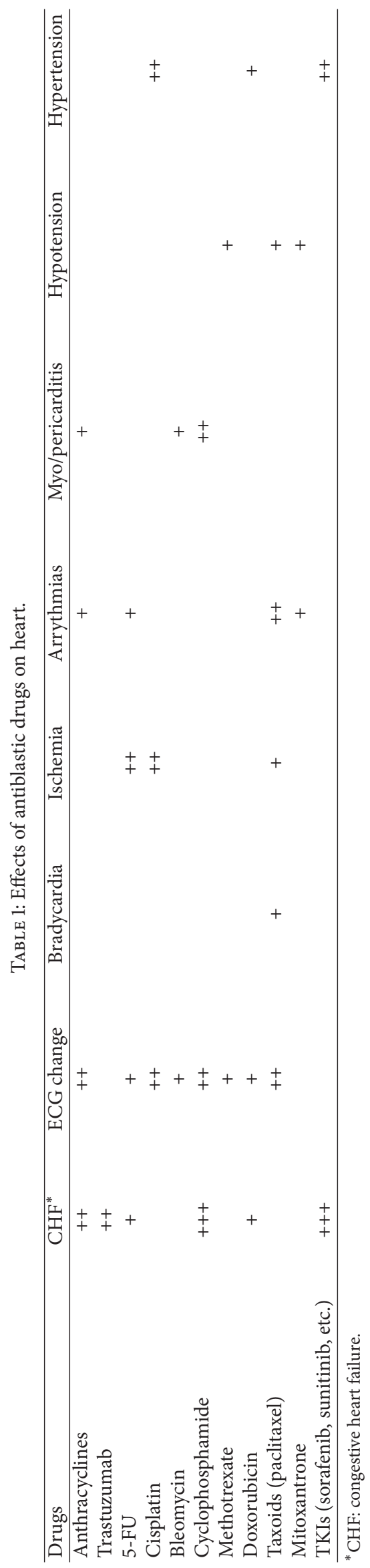


However a sudden atrial fibrillation was observed at the third week of chemotherapy administration in patients with myotonic dystrophy [7]. The clinical symptoms may include all signs of cardiomyopathy with electrophysiologic changes, decrease of left ventricular function, changes in exercisestress capacity, and overt signs of congestive heart failure [8]. During administration of taxoids, as paclitaxel, combined or with cisplatin, various cardiac disturbances, like bradyand tachyarrhythmias, atrioventricular and bundle branch blocks, and cardiac ischemia were reported [9]. Evidence of hypotension is also described, probably correlated to hypersensitivity reaction. A combination of doxorubicin and paclitaxel administration in rats is correlated to an increase of myocardial necrosis compared with those treated with DOX alone [10].

5-Fluorouracil (5-FU) has direct toxic effects on vascular endothelium that involves endothelial nitric oxide synthase and leads to coronary spasms and endothelium-independent vasoconstriction via protein kinase $C[11,12]$. Cardiotoxicity effects of 5-FU include cardiac arrhythmias, silent myocardial ischemia, angina, congestive heart failure, and even sudden death [13]. Various neoadjuvant chemoradiation therapies of squamous cell carcinoma are reported in the literature. They consisted of a combination between radiotherapy treatments and mitomycin-C and 5-fluorouracil [14]. A recent large meta-analysis shows that notwithstanding ongoing improvements in chemotherapy treatments, anthracyclines still represent a considerable risk of cardiotoxicity [15].

Other cytotoxic drugs that have been reported to be cardiotoxic are capecitabine, mitoxantrone, cisplatin, and newer drugs, like the monoclonal antibody trastuzumab or melphalan, fludarabine, mitomycin, busulfan, mechlorethamine and dacarbazine [16]. New generation of tyrosine kinase inhibitors (TKIs), like sorafenib and sunitinib, are associated with direct cardiotoxicity [17]. Since the antiblastic-induced cardiotoxicity is generally irreversible, it is crucial to detect the myocardial injury at its earliest possible stage; for this reason several experimental studies on cell cultures or animal models have been carried out.

Results on toxic effects of antiblastic drugs in various species were found extremely variable. Not only does the LD50 vary from species to species [18] but the qualitative character of the pharmacodynamic action of the drug also is equally varied [19]; therefore we can only partially compare precisely the dose and the toxic effects of antiblastic drugs between the model and the human animal model.

The major point of attack may be either the central nervous system or the heart. The rabbit is a representative animal showing cardiac responses [20], while in the dog [21] effects on central nervous system are the main response; rhesus monkey produces mixed-type responses [22]. However, despite the large number of investigations made, the results obtained in animal models are still hard to be translated to humans; therefore there is a critical need for continued translational research and animal studies to improve our understanding of the molecular mechanisms that underlie the cardiac dysfunction of antiblastic drugs.

\section{Mechanisms of Cardiotoxicity}

2.1. Role of Oxidative Stress. Antiblastic drugs, as most of xenobiotics, are generally metabolized through the NADPHcytochrome P450 system in order to increase their solubility in urine. In particular, doxorubicin could be substrate of several oxidoreductases like NADH-dehydrogenase of mitochondrial complex I and various cytoplasmic oxidoreductases, including xanthine oxidase. The oxidoreductive reaction starts with a single electron transfer from NADPH to doxorubicin forming a semiquinone radical that is complexed with iron ion in a ferrous form; this complex is responsible for the oxygen reduction, thus producing a superoxide ion [8]. The superoxide free radicals generated in mitochondria have cardiolipin as a preferential target. Cardiolipin is a major phospholipid component of the inner mitochondrial membrane and is required for the activity of respiratory chain. It is rich in polyunsaturated fatty acids and is particularly susceptible to peroxidative injury [23]; furthermore, evidence has been reported showing a strong affinity of doxorubicin for cardiolipin [24]. The drug-phospholipid complex formation leads to an inhibition of mitochondrial enzymes involved in oxidative phosphorylation. The mitochondrial membrane damage can also generate the inactivation of key transporters involved in ion homeostasis. Thus, the well-known cardiotoxicity of anthracycline could simply explain considering the fact that the cardiac tissue is rich in mitochondria. However, other factors are involved in anthracycline cardiotoxicity among which the relative lower amount of antioxidant defenses of heart compared with other tissues. It should be also considered that when the levels of free radicals increase, the apoptotic cascade is activated by cytochrome $\mathrm{c}$ being released from the damaged mitochondria, thus triggering apoptosis. Vásquez-Vivar et al. [25] have shown that doxorubicin binds to the reductase domain of endothelial nitric oxide synthase causing an increase in superoxide and a decrease in nitric oxide formation.

2.2. Role of Cytokines. Anthracyclines produce a drug-related systemic inflammation which has been found to be mediated by interleukins [26]. In particular, interleukin-1beta (IL1beta) has been implicated in this mechanism. Doxorubicin induces a systemic increase in IL-1beta and other inflammatory cytokines, chemokines, and growth factors including TNF-alpha, IL-6, CXCL1/Gro-alpha, CCL2/MCP-1, granulocyte colony stimulating factor, and CXCL10/IP-10. Studies on mice deficient in IL-1 receptor demonstrate that IL-1 signaling plays a role in the increase of IL- 6 and GCSF induced by doxorubicin. The IL-1beta release required the expression of caspase-1, NLRP3, and the adaptor protein ASC indicating that inflammation is mediated by the NLRP3 inflammasome. The molecular mechanisms by which anthracyclines trigger IL-1beta release are not completely understood; however the undesirable consequences of anthracyclines due to their inflammatory activity that complicate chemotherapy may be reduced by suppressing the actions of IL-1beta. It has been also showed that the administration of anthracyclines to mice having cancer stimulates the secretion of tumor necrosis factor alpha (TNF-alpha) in neoplastic tissue [27]. 
The antineoplastic effects of anthracyclines could be partially due to a local immune response that involves several distinct subsets of $\mathrm{T}$ lymphocytes and dendritic cells. However, the blockage of the TNF-alpha/TNF receptor system did not influence the antineoplastic effects of doxorubicin against MCA205 fibrosarcomas growing in C57BL/6 mice, F244 sarcomas developing in 129/Sv hosts, and H2N100 mammary carcinomas in $\mathrm{BALB} / \mathrm{c}$ mice. These findings show that, in contrast to other cytokines, TNF-alpha is not required to elicit anticancer immune responses. Aluise and coworkers [28] demonstrate that doxorubicin oxidizes plasma APOA1 that, in turn, enhances macrophage TNF-alpha release contributing to a possible TNF-alpha-mediated toxicity. Furthermore they produced evidence that reducing agent 2mercaptoethane sulfonate blocks this mechanism suggesting that this antioxidant could reduce systemic side effects of doxorubicin.

Doxorubicin has been also showed to be a potent inducer of apoptosis in both cardiomyocytes $\mathrm{H} 9 \mathrm{c} 2$ and osteosarcoma tumor cells U2OS; however, caspase activation and kinetics take place with significant differences between the two cell lines [29]. In fact, apoptosis is accompanied by relevant changes in levels of TNF-alpha receptor in H9c2 cardiomyocytes but not in U2OS cells. Moreover, treatment with exogenous TNF-alpha strongly increases the apoptotic effect of doxorubicin in $\mathrm{H} 9 \mathrm{c} 2$ cardiomyocytes but not in U2OS cells. The function of TNF receptors I and II is differently affected by doxorubicin which induces in $\mathrm{H} 9 \mathrm{c} 2$ cells an increase in the death domain-containing TNFR-1 protein levels and a decrease in the survival domain-containing TNFR-2 protein levels. These findings demonstrate a balance between proapoptotic and antiapoptotic signaling pathways in the cardiomyocyte survival after TNF stimulation showing a relevant role of TNF-alpha receptor-mediated signaling in cardiotoxicity induced by anthracyclines.

2.3. Calcium Homeostasis. Another aspect to be considered is the effect of anthracyclines on the role played by mitochondria in calcium homeostasis [30]. In fact the drug-induced malfunction of transporters involved in ion homeostasis can lead to a loss of mitochondrial calcium loading capacity which is observed in several in vitro and in vivo models $[31,32]$. Alterations in calcium transport can lead to tissue damage impairing the cardiac contraction. In vitro experiments demonstrate that doxorubicin treatment produces an irreversible decrease in mitochondrial calcium loading capacity. Moreover, anthracyclines could stimulate "in vitro" the release of calcium from isolated sarcoplasmic reticulum. In rodent models a decrease of calcium loading capacity together with alterations in cardiac mitochondrial function has been observed [33]. Verapamil, a calcium blocking agent, shows a protective effect against doxorubicin cardiotoxicity [34]. The antagonizing effect could be due to the ability of verapamil to inhibit the intracellular calcium overload. Contradictory results, however, arise from experiments showing an increase of cardiotoxicity when doxorubicin was given in combination with verapamil [35]. A possible explanation for this discrepancy could be due to the capacity of verapamil to inhibit the function of P-glycoprotein and therefore may increase intracellular cytotoxic drug concentrations. Other authors found that the additive cardiotoxicity of verapamil was due to its selective inhibition of cardiac actin gene [36], an effect which was also demonstrated with doxorubicin alone. Tagliaferri and coworkers [37] found side effects on heart electric conductance following infusion of high dose verapamil incorporated into cytotoxic chemotherapy. Several symptoms like premature ventricular beats, and mild and transient hypotension were observed. Hypokalemia was also detected probably as a consequence of transient activation of the renin-angiotensin system.

2.4. Metabolite Theory. To overcome the cardiotoxic effect of anthracyclines the use of antioxidants have been suggested $[38,39]$, however antioxidants has proven to be useful in delaying or preventing chronic cardiotoxicity in rodents [40] but not in dogs [41] or sheep [42]. For patients, contradictory results have been reported showing positive $[43,44]$ or no [45] effect. Taking into account these discrepancies, a new hypothesis has been made on the evidence that chronic cardiomyopathy develops after conversion of doxorubicin to the corresponding secondary alcohol metabolite doxorubicinol [46]. This metabolite is formed after the reduction of carbonyl group on the C-13 side chain; the reaction is probably mediated by cytoplasmic oxidoreductases [47]. The secondary alcohol metabolite production is suggested by several lines of evidence: (i) in rodents, after anthracycline treatment, a decline of cardiac function usually is observed when alcohol metabolite reachs its maximum levels in the heart [48]; (ii) overexpression of human carbonyl reductase in transgenic mice heart produces an accelerated development of cardiomyopathy [49]; (iii) modified anthracyclines with resistance to reduction of carbonyl moiety produce a less severe chronic cardiotoxicity in rats [50]. Due to their chemical structures, secondary alcohol metabolites are considerably less effective than their parent drugs at producing oxygen radicals, probably for their reduced affinity for quinone reductases [51]. However, secondary alcohol metabolites are several times more potent at inactivating membrane ATPases [52] and cytoplasmic aconitase/iron regulatory protein 1 [53]. The evidence that secondary alcohol metabolites can be involved in chronic cardiomyopathy suggested the hypothesis that the clinical use of anthracyclines could be improved by reducing their conversion to secondary alcohol. This goal could be reached in at least two ways: (i) a chemical modification of drugs to produce less alcohol metabolites and (ii) a development of inhibitors of reductases which are responsible for transformation of ketone/aldehyde moiety to alcohol. Obviously the investigations on the inhibitors have to consider possible differences in specificity and affinity between the reductases of humans and those of laboratory animals used to verify the protective efficacy of these inhibitors.

Recently [54] an effect of glutamine against oxidative damage due to doxorubicin has been reported. The free radicals produced by doxorubicin result in a decrease of 
glutathione (GSH) and a depletion of superoxide dismutase in cardiac muscle [55]. It seems that glutamine has a protective role in the myocardial cell by upregulating GSH and also by inducing the synthesis of heat shock protein 72 [56]. This protein is known to protect the myocardium against hypoxic/ischemic injury. Furthermore, the induction of heat shock protein 27 has been shown to be protective against cardiac injury induced by doxorubicin [57]. Glutamine also appears to be a potent inducer of myocardial HSP 72 in an in vivo rat model. Recently evidence has been produced indicating that glutamine can preserve the level of high-energy phosphate in myocardial tissue and prevent the stress-dependent accumulation of lactate, including ischemia/reperfusion injury [58].

It is now well assessed that anthracyclines possess the ability to bind covalently to DNA; the bind is strictly dependent on the availability of formaldehyde [59]. In fact, formaldehyde supplies the carbon required for the N-C$\mathrm{N}$ linkage necessary for the adduct formation with DNA. The resulting adduct is further stabilized by the formation of hydrogen bond with the complementary strand of DNA to crosslink the DNA duplex resulting in stabilization of the local region of DNA.

Doxorubicin is also known to intercalate itself into the DNA, with the inhibition of both DNA and RNA polymerase, thus blocking DNA replication and RNA transcription [60]. Recently it has been reported that doxorubicin is capable of intercalating with not only nuclear DNA but also mitochondrial DNA [61].

2.5. Tyrosine Kinase Inhibitors. Recent years have seen significant progress in cancer therapy through the development of "targeted therapies", in particular those using TKIs directed against certain tyrosine kinases whose abnormal activity triggers cancer development and progression through cell proliferation and neoangiogenesis. Multikinase inhibitors have been widely used alone and in combination with other drugs in cancer therapies for different tumor types $[62,63]$. Unfortunately, due to their large spectra of action, these inhibitors are also associated with toxicity to the heart $[64,65]$. For example, sunitinib inhibits a number of growth factor receptors regulating both tumor cell proliferation/survival and tumor angiogenesis including vascular endothelial growth factor receptors, platelet-derived growth factor receptors $\alpha$ and $\beta, \mathrm{c}$-Kit, FLT3, CSF1R, and RET [66]. However, care should be taken when cardiotoxicity in humans and animal models is compared. In fact it has been reported [67] that while the TKIs pazopanib, sunitinib and sorafenib, showed cardiotoxic effects in humans, studies in animal model failed to show cardiac toxicities for all of these TKIs. TKIs can be divided into two general classes: (i) humanized monoclonal antibodies directed against the tyrosine kinase receptor or their ligands and (ii) small molecules interacting with kinases inhibiting their activity. The use of both classes of TKIs revealed a relatively high rate of adverse cardiac events in the clinic, with systolic dysfunction and resultant heart failure as one of the most common and important side effects. TKIs are frequently used for the treatment of renal-cell carcinoma, gastrointestinal stromal tumors, and other tumor types in which these drugs are still under investigation. It seems that TKIs have as target AMPK which is a critical kinase controlling the balance between ATP and AMP levels [66]. Following conditions of energy stress, AMPK may act as a metabolic switch, increasing energy generation and inhibiting anabolic pathways. Studies on animals treated with sunitinib suggest that together with a potential misregulation in AMPK signaling a possible role is played by mitochondrial dysfunction leading to alterations in cardiac energy homeostasis. Most probably sunitinib induces a cardiac dysfunction that could be dependent on the simultaneous inhibition of multiple signaling pathways all of which are necessary for the preservation of cardiac function and which could play a pivotal role in the increased cardiac stress such as hypertension [68].

\section{Other Cardiotoxicity Mechanisms}

3.1. Taxoids. Paclitaxel is formulated in a cremophor EL vehicle to enhance the drug solubility and it is suggested that the vehicle and not the cytotoxic drug itself is responsible for the cardiac disturbances. However, the cardiac rhythm disturbances are not reported with use of other drugs containing cremophor EL such as cyclosporine $[69,70]$. The possible mechanism by which cremophor EL would cause cardiotoxicity is massive histamine release. Indeed, stimulation of histamine receptors in cardiac tissue in animal studies has resulted in conduction disturbances and arrhythmias. An alternative explanation for paclitaxel induced cardiotoxicity could be the induction of cardiac muscle damage by affecting subcellular organelles. Enhanced cardiac toxicity has been found in combined therapy of paclitaxel and doxorubicin. A similar effect has been shown for epirubicin. Docetaxel shows no increase in cardiac toxicity when combined with doxorubicin.

3.2. Cyclophosphamide and Ifosfamide. High dose cyclophosphamide is used in transplant regimens and is associated with acute cardiotoxicity such as cardiac decompensation as well as fatal cardiomyopathy. Acute reversible decrease in systolic function has been described. Ifosfamide cardiotoxicity is reported in only a single study. The pathogenesis is not fully understood but an increase in free oxygen radicals seems to play a role in oxazaphosphorine induced cardiotoxicity. This increase would be mediated by elevated intracellular levels of the actual cytotoxic metabolite phosphoramide mustard [71].

3.3. Cisplatin. Several factors have been suggested to be involved like vascular damage, alterations in platelet aggregation, and hypomagnesemia [72]. In experiments on animal platelets, cisplatin was able to trigger platelet aggregation and/or enhance thromboxane formation by platelets. Activation of an arachidonic pathway in platelets by cisplatin seemed to be involved [73].

3.4. Trastuzumab. Trastuzumab is a monoclonal antibody directed against the HER2 receptor protein on breast cancer 
cells and it has been used alone or in combination with other chemotherapeutic agents. Cardiac toxicity associated to trastuzumab seems to be similar with the congestive heart failure observed with anthracycline therapy [74].

\section{Concluding Remarks}

In the context of modern cancer chemotherapeutics, cancer survivors are living longer and being exposed to potential comorbidities related to noncancer side effects of such treatments as cardiotoxicity. These same toxic effects can also be detected in healthcare worker exposed during the manipulation of chemotherapy because several studies have identified the presence of drugs such as doxorubicin, epirubicin, cyclophosphamide, and 5-fluorouracil in these subjects. These side effects can be cause of severe morbidity and even mortality, so knowledge about their incidence and mechanism is important. The authors have reevaluated in the different articles available in the scientific literature the possible causes of cardiotoxicity due to administration of antiblastic drugs by using animal models. In fact, animal models have historically been unable to predict human response to drugs and this is the basis for their widespread use in human toxicity testing. The mechanisms of action described in the literature are different, such as, the oxidative stress for doxorubicin and misregulation in AMPK signaling by TKI. These results disclose a new scenario for prevention of heart complications.

We are now, in fact, able to identify specific early biomarker of chemotherapy cardiotoxicity, discovered on animal models, and to develop supportive therapies to reduce or eliminate the appearance of these side effects in humans.

\section{Conflict of Interests}

The authors declare that there is no conflict of interests regarding the publication of this paper.

\section{References}

[1] T. H. Connor, G. DeBord, J. R. Pretty et al., "Evaluation of antineoplastic drug exposure of health care workers at three university-based US cancer centers," Journal of Occupational and Environmental Medicine, vol. 52, no. 10, pp. 1019-1027, 2010.

[2] M. Pieri, L. Castiglia, P. Basilicata, N. Sannolo, A. Acampora, and N. Miraglia, "Biological monitoring of nurses exposed to doxorubicin and epirubicin by a validated liquid chromatography/fluorescence detection method," The Annals of Occupational Hygiene, vol. 54, no. 4, pp. 368-376, 2010.

[3] K. J. Schimmel, D. J. Richel, R. B. van den Brink, and H. J. Guchelaar, "Cardiotoxicity of cytotoxic drugs," Cancer Treatment Reviews, vol. 30, no. 2, pp. 181-191, 2004.

[4] Y. J. Kang, "Molecular and cellular mechanisms of cardiotoxicity," Environmental Health Perspectives, vol. 109, supplement 1, pp. 27-34, 2001.

[5] H. Broder, R. A. Gottlieb, and N. E. Lepor, "Chemotherapy and cardiotoxicity," Reviews in Cardiovascular Medicine, vol. 9, no. 2, pp. 75-83, 2008.
[6] C. Dechant, M. Baur, R. Bock et al., "Acute reversible heart failure caused by coronary vasoconstriction due to continuous 5-fluorouracil combination chemotherapy," Case Reports in Oncology, vol. 5, no. 2, pp. 296-301, 2012.

[7] L. Montella, M. Caraglia, R. Addeo et al., "Atrial fibrillation following chemotherapy for stage IIIE diffuse large B-cell gastric lymphoma in a patient with myotonic dystrophy (Steinert's disease)," Annals of Hematology, vol. 84, no. 3, pp. 192-193, 2005.

[8] F. S. Carvalho, A. Burgeiro, R. Garcia, A. J. Moreno, R. A. Carvalho, and P. J. Oliveira, "Doxorubicin-induced cardiotoxicity: from bioenergetic failure and cell death to cardiomyopathy," Medicinal Research Reviews, vol. 34, no. 1, pp. 106-135, 2014.

[9] J. B. Vermorken, "The integration of paclitaxel and new platinum compounds in the treatment of advanced ovarian cancer," International Journal of Gynecological Cancer, vol. 11, supplement s1, pp. 21-30, 2001.

[10] S. Y. Saad, T. A. Najjar, and M. Alashari, "Cardiotoxicity of doxorubicin/paclitaxel combination in rats: effect of sequence and timing of administration," Journal of Biochemical and Molecular Toxicology, vol. 18, no. 2, pp. 78-86, 2004.

[11] M. Lamberti, M. Caraglia, S. Zappavigna et al., "Evaluation in vitro of the cardiotoxic effects of 5-fluorouracil for the prevention of cardiovascular damage in health workers occupationally exposed," Giornale Italiano di Medicina del Lavoro ed Ergonomia, vol. 33, no. 3, supplement, pp. 298-302, 2011.

[12] M. Lamberti, S. Porto, M. Marra et al., "5-Fluorouracil induces apoptosis in rat cardiocytes through intracellular oxidative stress," Journal of Experimental \& Clinical Cancer Research, vol. 31, article 60, 2012.

[13] B. B. Hasinoff, "The cardiotoxicity and myocyte damage caused by small molecule anticancer tyrosine kinase inhibitors is correlated with lack of target specificity," Toxicology and Applied Pharmacology, vol. 244, no. 2, pp. 190-195, 2010.

[14] M. Indinnimeo, A. Impagnatiello, G. D’Ettorre et al., "BuschkeLöwenstein tumor with squamous cell carcinoma treated with chemo-radiation therapy and local surgical excision: report of three cases," World Journal of Surgical Oncology, vol. 11, article 231, 2013.

[15] M. Lotrionte, G. Biondi-Zoccai, A. Abbate et al., "Review and meta-analysis of incidence and clinical predictors of anthracycline cardiotoxicity," The American Journal of Cardiology, vol. 112, no. 12, pp. 1980-1984, 2013.

[16] V. Brower, "Cardiotoxicity debated for anthracyclines and trastuzumab in breast cancer," Journal of the National Cancer Institute, vol. 105, no. 12, pp. 835-836, 2013.

[17] V. Chintalgattu, M. L. Rees, J. C. Culver et al., "Coronary microvascular pericytes are the cellular target of sunitinib malate-induced cardiotoxicity," Science Translational Medicine, vol. 5, no. 187, p. 187ra69, 2013.

[18] M. Wasowska, I. Oszczapowicz, J. Wietrzyk et al., "Influence of the structure of new anthracycline antibiotics on their biological properties," Anticancer Research, vol. 25, no. 3, pp. 2043-2048, 2005.

[19] R. Danesi, S. Fogli, A. Gennari, P. Conte, and M. Del Tacca, "Pharmacokinetic-pharmacodynamic relationships of the anthracycline anticancer drugs," Clinical Pharmacokinetics, vol. 41, no. 6, pp. 431-444, 2002.

[20] E. Jirkovský, O. Lenčová-Popelová, M. Hroch et al., "Early and delayed cardioprotective intervention with dexrazoxane each show different potential for prevention of chronic anthracycline cardiotoxicity in rabbits," Toxicology, vol. 311, no. 3, pp. 191-204, 2013. 
[21] S. Gillings, J. Johnson, A. Fulmer, and M. Hauck, "Effect of a 1-hour IV infusion of doxorubicin on the development of cardiotoxicity in dogs as evaluated by electrocardiography and echocardiography," Veterinary Therapeutics, vol. 10, no. 1-2, pp. 46-58, 2009.

[22] D. Beulz-Riche, J. Robert, C. Menard, and D. Ratanasavanh, "Metabolism of methoxymorpholino-doxorubicin in rat, dog and monkey liver microsomes: comparison with human microsomes," Fundamental and Clinical Pharmacology, vol. 15, no. 6, pp. 373-378, 2001.

[23] M. Tokarska-Schlattner, M. Dolder, I. Gerber, O. Speer, T. Wallimann, and U. Schlattner, "Reduced creatine-stimulated respiration in doxorubicin challenged mitochondria: particular sensitivity of the heart," Biochimica et Biophysica Acta, vol. 1767, no. 11, pp. 1276-1284, 2007.

[24] M. A. Parker, V. King, and K. P. Howard, "Nuclear magnetic resonance study of doxorubicin binding to cardiolipin containing magnetically oriented phospholipid bilayers," Biochimica et Biophysica Acta, vol. 1514, no. 2, pp. 206-216, 2001.

[25] J. Vásquez-Vivar, P. Martasek, N. Hogg, B. S. Masters, K. A. Pritchard Jr., and B. Kalyanaraman, "Endothelial nitric oxide synthase-dependent superoxide generation from adriamycin," Biochemistry, vol. 36, no. 38, pp. 11293-11297, 1997.

[26] K. A. Sauter, L. J. Wood, J. Wong, M. Iordanov, and B. E. Magun, "Doxorubicin and daunorubicin induce processing and release of interleukin- $1 \beta$ through activation of the NLRP3 inflammasome," Cancer Biology and Therapy, vol. 11, no. 12, pp. 1008-1016, 2011.

[27] Y. Ma, T. Yamazaki, H. Yang et al., "Tumor necrosis factor is dispensable for the success of immunogenic anticancer chemotherapy," Oncoimmunology, vol. 2, no. 6, Article ID e24786, 2013.

[28] C. D. Aluise, S. Miriyala, T. Noel et al., "2-Mercaptoethane sulfonate prevents doxorubicin-induced plasma protein oxidation and TNF- $\alpha$ release: implications for the reactive oxygen speciesmediated mechanisms of chemobrain," Free Radical Biology and Medicine, vol. 50, no. 11, pp. 1630-1638, 2011.

[29] E. Chiosi, A. Spina, A. Sorrentino et al., "Change in TNF- $\alpha$ receptor expression is a relevant event in doxorubicin-induced H9c2 cardiomyocyte cell death," Journal of Interferon and Cytokine Research, vol. 27, no. 7, pp. 589-597, 2007.

[30] H. A. Gambliel, B. E. Burke, B. J. Cusack et al., "Doxorubicin and C-13 deoxydoxorubicin effects on ryanodine receptor gene expression," Biochemical and Biophysical Research Communications, vol. 291, no. 3, pp. 433-438, 2002.

[31] M. Verma, N. Shulga, and J. G. Pastorino, "Sirtuin-4 modulates sensitivity to induction of the mitochondrial permeability transition pore," Biochimica et Biophysica Acta, vol. 1827, no. 1, pp. 38-49, 2013.

[32] A. Ascensão, J. Lumini-Oliveira, N. G. Machado et al., "Acute exercise protects against calcium-induced cardiac mitochondrial permeability transition pore opening in doxorubicintreated rats," Clinical Science, vol. 120, no. 1, pp. 37-49, 2011.

[33] C. Richard, S. Ghibu, S. Delemasure-Chalumeau et al., "Oxidative stress and myocardial gene alterations associated with doxorubicin-induced cardiotoxicity in rats persist for 2 months after treatment cessation," The Journal of Pharmacology and Experimental Therapeutics, vol. 339, no. 3, pp. 807-814, 2011.

[34] F. Rossi, W. Filippelli, S. Russo, A. Filippelli, and L. Berrino, "Cardiotoxicity of doxorubicin: effects of drugs inhibiting the release of vasoactive substances," Pharmacology and Toxicology, vol. 75, no. 2, pp. 99-107, 1994.
[35] T. Budde, J. Haney, S. Bien et al., "Acute exposure to doxorubicin results in increased cardiac P-glycoprotein expression," Journal of Pharmaceutical Sciences, vol. 100, no. 9, pp. 3951-3958, 2011.

[36] H. Akimoto, N. A. Bruno, D. L. Slate, M. E. Billingham, S. V. Torti, and F. M. Torti, "Effect of verapamil on doxorubicin cardiotoxicity: altered muscle gene expression in cultured neonatal rat cardiomyocytes," Cancer Research, vol. 53, no. 19, pp. 46584664, 1993

[37] P. Tagliaferri, P. Correale, M. Mottola et al., "Cardiovascular monitoring of drug-resistant lymphoma patients treated with epoch chemotherapy plus high dose verapamil in continuousinfusion," Oncology Reports, vol. 1, no. 2, pp. 341-344, 1994.

[38] H. Jacobs, R. Peters, G. J. den Hartog, W. J. van der Vijgh, A. Bast, and G. R. Haenen, "Identification of the metabolites of the antioxidant flavonoid 7-mono-O-( $\beta$-hydroxyethyl)-rutoside in mice," Drug Metabolism and Disposition, vol. 39, no. 5, pp. 750756, 2011.

[39] S. Arunachalam, S. Y. Kim, S. H. Lee et al., "Davallialactone protects against adriamycin-induced cardiotoxicity in vitro and in vivo," Journal of Natural Medicines, vol. 66, no. 1, pp. 149-157, 2012.

[40] A. M. Osman, S. E. Al-Harthi, O. M. Alarabi et al., "Chemosensetizing and cardioprotective effects of resveratrol in doxorubicin-treated animals," Cancer Cell International, vol. 13, article 52, 2013.

[41] E. H. Herman, V. J. Ferrans, C. E. Myers, and J. F. Van Vleet, "Comparison of the effectiveness of (+/-)-1,2bis(3,5-dioxopiperazinyl-1-yl)propane (ICRF-187) and Nacetylcysteine in preventing chronic doxorubicin cardiotoxicity in beagles," Cancer Research, vol. 45, no. 1, pp. 276-281, 1985.

[42] A. Carbone, P. J. Psaltis, A. J. Nelson et al., "Dietary omega-3 supplementation exacerbates left ventricular dysfunction in an ovine model of anthracycline-induced cardiotoxicity," Journal of Cardiac Failure, vol. 18, no. 6, pp. 502-511, 2012.

[43] A. Bast, G. R. Haenen, A. M. Bruynzeel, and W. J. van der Vijgh, "Protection by flavonoids against anthracycline cardiotoxicity: from chemistry to clinical trials," Cardiovascular Toxicology, vol. 7, no. 2, pp. 154-159, 2007.

[44] C. D. Aluise, D. St Clair, M. Vore, and D. A. Butterfield, "In vivo amelioration of adriamycin induced oxidative stress in plasma by gamma-glutamylcysteine ethyl ester (GCEE)," Cancer Letters, vol. 282, no. 1, pp. 25-29, 2009.

[45] C. Myers, R. Bonow, S. Palmeri et al., "A randomized controlled trial assessing the prevention of doxorubicin cardiomyopathy by $\mathrm{N}$-acetylcysteine," Seminars in Oncology, vol. 10, no. 1, supplement 1, pp. 53-55, 1983.

[46] G. Minotti, S. Licata, A. Saponiero et al., "Anthracycline metabolism and toxicity in human myocardium: comparisons between doxorubicin, epirubicin, and a novel disaccharide analogue with a reduced level of formation and [4Fe-4S] reactivity of its secondary alcohol metabolite," Chemical Research in Toxicology, vol. 13, no. 12, pp. 1336-1341, 2000.

[47] O. S. Bains, M. J. Karkling, T. A. Grigliatti, R. E. Reid, and K. W. Riggs, "Two nonsynonymous single nucleotide polymorphisms of human carbonyl reductase 1 demonstrate reduced in vitro metabolism of daunorubicin and doxorubicin," Drug Metabolism and Disposition, vol. 37, no. 5, pp. 1107-1114, 2009.

[48] P. S. Mushlin, B. J. Cusack, R. J. Boucek Jr., T. Andrejuk, X. Li, and R. D. Olson, "Time-related increases in cardiac concentrations of doxorubicin could interact with doxorubicin to depress myocardial contractile function," British Journal of Pharmacology, vol. 110, no. 3, pp. 975-982, 1993. 
[49] G. L. Forrest, B. Gonzalez, W. Tseng, X. Li, and J. Mann, "Human carbonyl reductase overexpression in the heart advances the development of doxorubicin-induced cardiotoxicity in transgenic mice," Cancer Research, vol. 60, no. 18, pp. 5158-5164, 2000.

[50] R. Cirillo, G. Sacco, S. Venturella, J. Brightwell, A. Giachetti, and S. Manzini, "Comparison of doxorubicin-and MEN 10755induced long-term progressive cardiotoxicity in the rat," Journal of Cardiovascular Pharmacology, vol. 35, no. 1, pp. 100-108, 2000.

[51] P. G. Gervasi, M. R. Agrillo, A. Lippi, N. Bernardini, R. Danesi, and M. Del Tacca, "Superoxide anion production by doxorubicin analogs in heart sarcosomes and by mitochondrial NADH dehydrogenase," Research Communications in Chemical Pathology and Pharmacology, vol. 67, no. 1, pp. 101-115, 1990.

[52] R. J. Boucek Jr., R. D. Olson, D. E. Brenner, E. M. Ogunbunmi, M. Inui, and S. Fleischer, "The major metabolite of doxorubicin is a potent inhibitor of membrane-associated ion pumps: a correlative study of cardiac muscle with isolated membrane fractions," The Journal of Biological Chemistry, vol. 262, no. 33, pp. 15851-15856, 1987.

[53] G. Minotti, R. Ronchi, E. Salvatorelli, P. Menna, and G. Cairo, "Doxorubicin irreversibly inactivates iron regulatory proteins 1 and 2 in cardiomyocytes: evidence for distinct metabolic pathways and implications for iron-mediated cardiotoxicity of antitumor therapy," Cancer Research, vol. 61, no. 23, pp. 84228428, 2001.

[54] K. Gaurav, R. K. Goel, M. Shukla, and M. Pandey, "Glutamine: a novel approach to chemotherapy-induced toxicity," Indian Journal of Medical and Paediatric Oncology, vol. 33, no. 1, pp. 13-20, 2012.

[55] S. I. Glushkov, S. A. Kutsenko, T. M. Novikova, and V. V. Axenov, "Glutathione metabolism in rat cardiac tissues in acute doxorubicin-induced intoxication," Voprosy Onkologii, vol. 51, no. 1, pp. 108-112, 2005.

[56] H. Xue, D. Slavov, and P. E. Wischmeyer, "Glutamine-mediated dual regulation of heat shock transcription factor-1 activation and expression," The Journal of Biological Chemistry, vol. 287, no. 48, pp. 40400-40413, 2012.

[57] L. Liu, X.-J. Zhang, B. Qian, X.-Y. Min, and Y.-L. Cheng, "Heat shock protein 27 attenuated doxorubicin-induced myocardial damage by reducing cardiomyocyte apoptosis, mitochondria damage and protein carbonylation," Zhonghua Xin Xue Guan Bing Za Zhi, vol. 36, no. 11, pp. 1021-1026, 2008.

[58] P. E. Wischmeyer, D. Jayakar, U. Williams et al., "Single dose of glutamine enhances myocardial tissue metabolism, glutathione content, and improves myocardial function after ischemiareperfusion injury," Journal of Parenteral and Enteral Nutrition, vol. 27, no. 6, pp. 396-403, 2003.

[59] R. A. Forrest, L. P. Swift, B. J. Evison et al., "The hydroxyl epimer of doxorubicin controls the rate of formation of cytotoxic anthracycline-DNA adducts," Cancer Chemotherapy and Pharmacology, vol. 71, no. 3, pp. 809-816, 2013.

[60] O. Tacar, P. Sriamornsak, and C. R. Dass, "Doxorubicin: an update on anticancer molecular action, toxicity and novel drug delivery systems," Journal of Pharmacy and Pharmacology, vol. 65, no. 2, pp. 157-170, 2013.

[61] N. Ashley and J. Poulton, "Mitochondrial DNA is a direct target of anti-cancer anthracycline drugs," Biochemical and Biophysical Research Communications, vol. 378, no. 3, pp. 450455, 2009.
[62] C. F. Greineder, S. Kohnstamm, and B. Ky, "Heart failure associated with sunitinib: lessons learned from animal models," Current Hypertension Reports, vol. 13, no. 6, pp. 436-441, 2011.

[63] M. Caraglia, G. Giuberti, M. Marra et al., "Oxidative stress and ERK1/2 phosphorylation as predictors of outcome in hepatocellular carcinoma patients treated with sorafenib plus octreotide LAR," Cell Death and Disease, vol. 2, no. 4, article e150, 2011.

[64] A. Y. Khakoo, P. P. Liu, T. Force et al., "Cardiotoxicity due to cancer therapy," Texas Heart Institute Journal, vol. 38, no. 3, pp. 253-256, 2011.

[65] T. Force, D. S. Krause, and R. A. Van Etten, "Molecular mechanisms of cardiotoxicity of tyrosine kinase inhibition," Nature Reviews Cancer, vol. 7, no. 5, pp. 332-344, 2007.

[66] R. Kerkela, K. C. Woulfe, J.-B. Durand et al., "Sunitinib-induced cardiotoxicity is mediated by off-target inhibition of AMPactivated protein kinase," Clinical and Translational Science, vol. 2, no. 1, pp. 15-25, 2009.

[67] B. Yang and T. Papoian, "Tyrosine kinase inhibitor (TKI)induced cardiotoxicity: approaches to narrow the gaps between preclinical safety evaluation and clinical outcome," Journal of Applied Toxicology, vol. 32, no. 12, pp. 945-951, 2012.

[68] D. Hannani, C. Locher, T. Yamazaki et al., "Contribution of humoral immune responses to the antitumor effects mediated by anthracyclines," Cell Death and Differentiation, vol. 21, no. 1, pp. 50-58, 2014.

[69] E. Senkus and J. Jassem, "Cardiovascular effects of systemic cancer treatment," Cancer Treatment Reviews, vol. 37, no. 4, pp. 300-311, 2011.

[70] A. Pieniążek, J. Czepas, J. Piasecka-Zelga, K. Gwoździński, and A. Koceva-Chyła, "Oxidative stress induced in rat liver by anticancer drugs doxorubicin, paclitaxel and docetaxel," Advances in Medical Sciences, vol. 58, no. 1, pp. 104-111, 2013.

[71] K. Kandylis, M. Vassilomanolakis, S. Tsoussis, and A. P. Efremidis, "Ifosfamide cardiotoxicity in humans," Cancer Chemotherapy and Pharmacology, vol. 24, no. 6, pp. 395-396, 1989.

[72] J. Nuver, E. C. De Haas, Z. M. Van, J. A. Gietema, and C. Meijer, "Vascular damage in testicular cancer patients: a study on endothelial activation by bleomycin and cisplatin in vitro," Oncology Reports, vol. 23, no. 1, pp. 247-253, 2010.

[73] F. Zhang, G. Suarez, J. Sha, J. C. Sierra, J. W. Peterson, and A. K. Chopra, "Phospholipase A2-activating protein (PLAA) enhances cisplatin-induced apoptosis in HeLa cells," Cellular Signalling, vol. 21, no. 7, pp. 1085-1099, 2009.

[74] M. Bonifazi, M. Franchi, M. Rossi et al., "Trastuzumab-related cardiotoxicity in early breast cancer: a cohort study," The Oncologist, vol. 18, no. 7, pp. 795-801, 2013. 


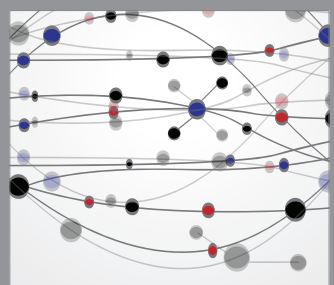

The Scientific World Journal
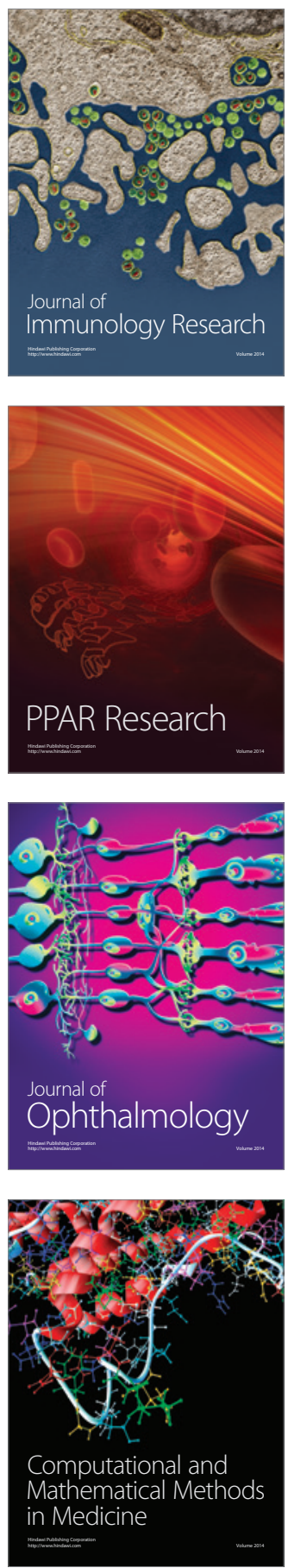

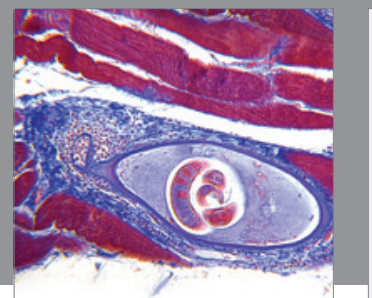

Gastroenterology

Research and Practice
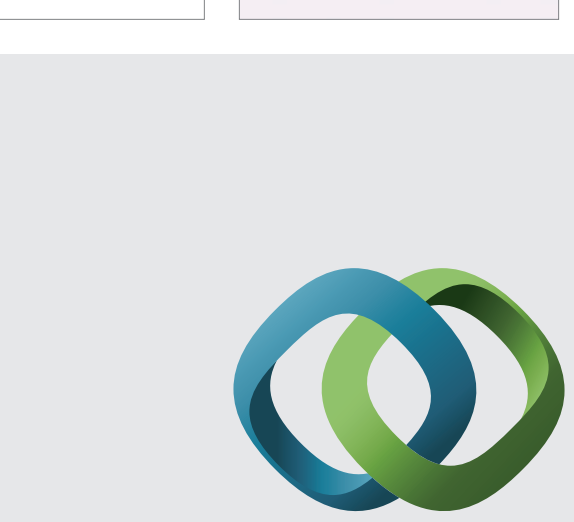

\section{Hindawi}

Submit your manuscripts at

http://www.hindawi.com
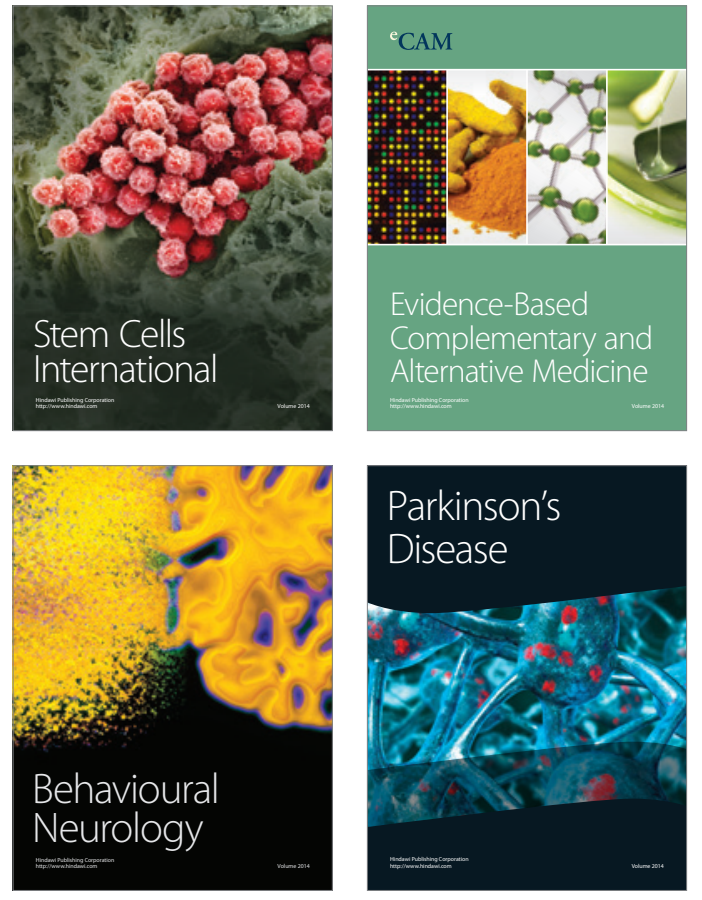
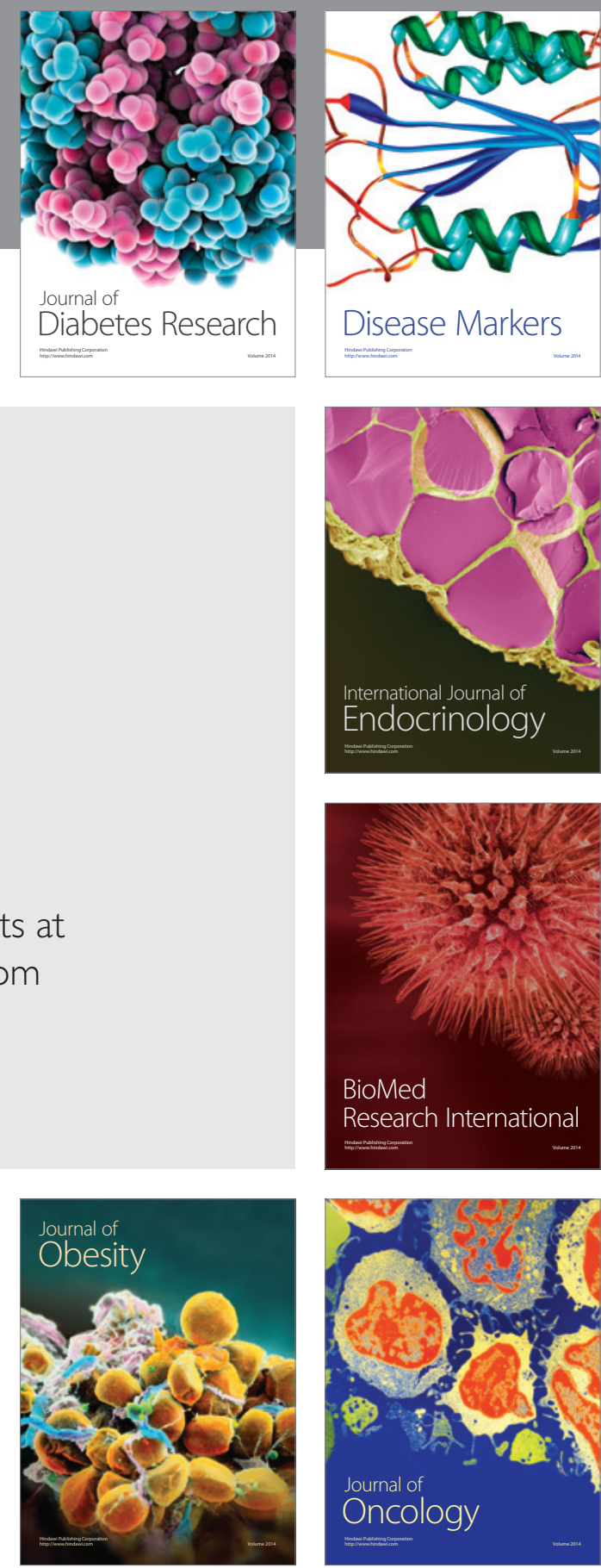

Disease Markers
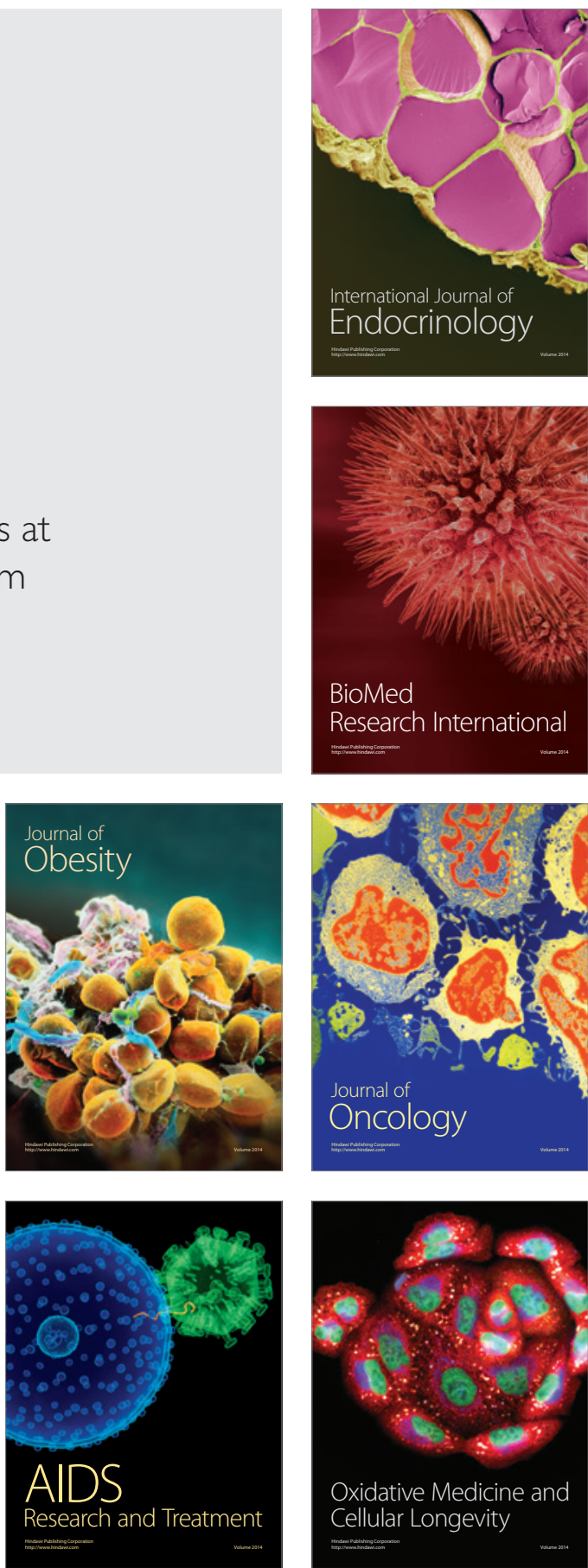